\title{
0 \\ Microplastics (MPs) as a Chemical Time Bomb: The New Emerging Silent Menace to Public Health
}

IJCRR

Section: Healthcare

ISI Impact Factor

(2019-20): 1.628
IC Value (2019): 90.81

$\operatorname{SIIF}(2020)=7.893$

\author{
Tomy M. Joseph \\ Department of Polymer Technology, Chemical Faculty, Gdansk University of Technology, Gdansk, Poland
}

\section{INTRODUCTION}

One of the numerous aspects of plastic contamination is microplastics (MPs). It may contain a variety of pollutants, including trace metals and certain organic compounds that are potentially hazardous. Once in the body, these compounds may leak off the plastic surface, raising the risk of harmful consequences. MPs have the potential to cause cancer due to their carcinogenic characteristics. Under the impact of sunshine, heat, wind, and pressure, the plastics we leave behind disintegrate into smaller particles. They can move from one location to another by air and water because of their tiny weight and size. As a result, they are everywhere and may be found in the air we breathe, the water we drink, and the food we consume, regardless of where they came from. MPs are derived from man-made components. Engineered MP polymers utilized as components for many common products and MPs that disintegrate into huge plastic materials are the two primary ways they appear in nature. These are referred to as main and secondary sources, respectively. MP particles have been discovered to be more abundant in oceans than in other areas of the environment. According to studies, the quantity of MP in seawater has risen five-fold in the last ten years. MPs have been discovered in significant amounts in soils, including up to $7 \%$ in agriculture. Different forms, sizes, colors, and chemical compositions are found in MP particles. MPs are particles with a diameter of less than 5 $\mathrm{mm}$. Nanoparticles are those with a diameter of less than 100 $\mathrm{nm}$. Nanoplastics (NPs) are polymers that are smaller than a $\mu \mathrm{m}$, according to some experts. MPs are thought to have a diameter of between $100 \mathrm{~nm}$ to $5 \mathrm{~mm}$. They appear as long threads and thin, spherical or other crystals.

\section{ENVIRONMENTAL AND HEALTHCARE IMPACTS}

Due to natural biological processes, water filtering, and agricultural operations, MPs mix with the soil structure as they reach the soil. As a result, bio-inorganic, organic, and inor- ganic components in soil and water react differently, resulting in a variety of chemical and physical changes. Very tiny MPs enter live creatures' bodies via the airways and food, affecting critical processes. Microplankton, copypods, bivalves, worms, and even big fish and birds have been discovered to have MPs. They reach a large number of people via the food chain of high-ranking organisms. MPs have also been shown to have a negative impact on the health of these organisms in studies. Through food, air, and water, MPs reach not just marine life but also live creatures, including people. It has been discovered that they have a negative impact on the development of earthworms and plants. Many species, both vertebrate and non-vertebrate, have stunted development and decreased fertility as a result of MPs. It obstructs the digestive and respiratory systems, causing harm. It also has an impact on enzyme and hormone synthesis and function. MPs have been found to have a variety of effects on plant development and metabolism. In the presence of MP polymers, seed germination rate, root, and stem development, and chlorophyll levels were found to be substantially decreased. Large quantities of $\mathrm{CO}_{2}$, in the form of MP particles, have been discovered on the ocean bottom, according to studies. MPs' waterproofing surface is readily adhered to by polyaromatic hydrocarbons with hydrophobicity. Hazardous chemicals are transported with MPs in this manner. As a result, these compounds have the potential to spread unnaturally. MPs have been shown to hasten the migration of harmful heavy metals and polychlorinated biphenyls into seawater in investigations. Some hazardous bacteria and algae have been discovered to adhere to the surface of MP particles and travel great distances in this manner.

\section{MOLECULAR AND CELLULAR MECHANISMS OF PLASTIC MICROMATERIAL / NANOMATERIAL DAMAGE AND DISEASE}

MPs and/or NPs may enter the bloodstream through trophic transfer from the gut or via the air. MPs or NPs block the efflux pump and cause cytotoxicity in human intestinal cells. 
The cytotoxicity caused by MPs and/or NPs cause oxidative stress by generating free radicals from reactive oxygen species (ROS). This link has been discovered in monogonont rotifer, Caenorhabditis elegans, Danio rerio, mouse liver, and human intestinal cell lines, according to many investigations. By modulating antioxidant mechanisms, excessive ROS may disrupt cell homeostasis. The antioxidants generated in reaction to damage to cellular components such as DNA, carbohydrates, lipids, and proteins are overwhelmed by ROS. Gene instability, physiological changes, and carcinogenesis are all linked to this damage. Pocillopora damicornis, a scleractinian coral, was exposed to MPs and showed enhanced superoxide dismutase (SOD) and catalase (CAT) activity, as well as glutathione- $S$-transferase (GST) and alkaline phosphatase (ALP) function loss. In coral, antioxidant enzymes SOD and CAT, detoxifying enzyme GST, and immunological enzyme ALP all play a role. MPs also have a role in the stress response, zymogen granules, the c-Jun N-terminal kinase signaling pathways, sterol transport, and the epidermal growth factor-extracellular signal-regulated kinase $1 / 2$ pathway in coral. In Caenorhabditis elegans, PS NPs also enhance oxidative stress, activate genes in the nuclear factor $\mathrm{E}_{2}$-related factor signaling pathway, and increase GST enzyme expression. PS MPs also cause inflammation and activate SOD and CAT activity in the livers of Danio rerio and mice, according to a prior study. These results suggest that the primary mechanism of toxicity induction in these species is oxidative stress. MPs may influence amino acid metabolism by raising arginine and tyrosine, and bile acid metabolism by regulating taurocholic acid (TCA), $\beta$-muricholic acid ( $\beta \mathrm{MCA})$, ATP-binding cassette, subfamily B, member 11 (Abcb11), and cholesterol 7a-hydroxylase levels (Cyp7a1). They also change the amounts of triglycerides, total cholesterol $(\mathrm{TCH})$, and pyruvate in the liver, which affects lipid metabolism. In a mouse model, PS MPs enhanced acetylcholinesterase activity and associated neurotransmitters including threonine, aspartate, and taurine. MPs and NPs can trigger immune responses, change gene expression, and cause genotoxicity. VC increases the expression of fibrosis-related proteins including CTGF, PAI-1, and collagen-1, as well as autophagy-related proteins like Beclin-1 and LC3-II in kidney cells. Angiosarcoma of the liver is caused by VC, which is a carcinogenic agent. $\mathrm{VC}$ has been linked to a variety of DNA changes, including Ras mutations, Kras-2 mutations, p53 mutations, and p21 mutations, according to research.

\section{SOLUTIONS FOR REDUCING PLASTIC MICROMATERIALS AND NANOMATERIALS}

Political entities all around the globe have made recommendations for prevention and cleanup, such as Africa's plastic reduction strategy, which ranks first in the world. The Ocean Cleanup, headquartered in the Netherlands, employs enormous drift nets to decrease the size of the Great Pacific Garbage Patch. MP particles have been discovered in many nations' wastewater treatment facilities. Filters on big drains in Australia prevent trash from entering the ocean. One of the components of this huge area of plastic waste is single-use plastic products. Since October 2, 2019, single-use plastic goods such as plastic bags, plastic spoons, plastic cups, plastic drinking straws, plastic jars, and plastic bottles have been prohibited in India. By 2021, the EU has established a goal of eliminating certain single-use plastic products. Single-use plastic products such as plastic straws are being phased out, with companies developing a reed pipe to replace plastic straws and developing a sugar cane straw. In addition, several nations have prohibited face cleansers that contain MP particles. Plastic trash has been converted into resources on the one hand. For instance, a firm in the Netherlands utilizes plastic to replace conventional road materials, and it is $60 \%$ stronger than asphalt. In India, abandoned fishing nets have been converted into surfboards; in the UK, students have successfully developed plastic replacements using fish skin and red algae as raw materials; and in Mexico, scientists have developed nontoxic edible plastic using cactus fruit. Plastics, on the other hand, form MPs and NPs as a result of physical and chemical modifications. As a result, microbial biodegradation may be utilized to break down the polymers into smaller monomers. When plastics break down monomers into $\mathrm{CO}_{2}$ and water, biodegradation is effective. Marine microorganisms may be used to aid in the biodegradation of plastic trash. PS is known to breakdown in yellow mealworms' guts. PVC, PHB, and PLA are among the polymers that many fungus strains may destroy. Several enzymes have recently been discovered to be capable of decomposing PET polymers. 